\title{
Defective Respiratory Rhythmogenesis and Loss of Central Chemosensitivity in Phox2b Mutants Targeting Retrotrapezoid Nucleus Neurons
}

\author{
Véronique Dubreuil, ${ }^{1,2 \star}$ Muriel Thoby-Brisson, ${ }^{3 \star}$ Murielle Rallu, ${ }^{1,2}$ Karin Persson, ${ }^{3}$ Alexandre Pattyn, ${ }^{1,2}$ \\ Carmen Birchmeier, ${ }^{4}$ Jean-François Brunet, ${ }^{1,2}$ Gilles Fortin, ${ }^{3}$ and Christo Goridis ${ }^{1,2}$ \\ ${ }^{1}$ Département de Biologie, Ecole normale supérieure, 75005 Paris, France, ${ }^{2}$ Centre National de la Recherche Scientifique (CNRS), Unité Mixte de Recherche \\ 8542, 75005 Paris, France, ${ }^{3}$ Neurobiologie Génétique et Intégrative, Institut de Neurobiologie Alfred Fessard, CNRS Unité Propre de Recherche 2216,91198 \\ Gif sur Yvette, France, and ${ }^{4}$ Max Delbrück Center for Molecular Medicine, 13125 Berlin, Germany
}

The retrotrapezoid nucleus (RTN) is a group of neurons in the rostral medulla, defined here as Phox2b-, Vglut2-, neurokinin1 receptor-, and Atoh1-expressing cells in the parafacial region, which have been proposed to function both as generators of respiratory rhythm and as central respiratory chemoreceptors. The present study was undertaken to assess these two putative functions using genetic tools. We generated two conditional Phox $2 b$ mutations, which target different subsets of Phox $2 b$-expressing cells, but have in common a massive depletion of RTN neurons. In both conditional mutants as well as in the previously described Phox $2 b^{27 A l a}$ mutants, in which the RTN is also compromised, the respiratory-like rhythmic activity normally seen in the parafacial region of fetal brainstem preparations was completely abrogated. Rhythmic motor bursts were recorded from the phrenic nerve roots in the mutants, but their frequency was markedly reduced. Both the rhythmic activity in the RTN region and the phrenic nerve discharges responded to a low pH challenge in control, but not in the mutant embryos. Together, our results provide genetic evidence for the essential role of the Phox $2 \mathrm{~b}$-expressing RTN neurons both in establishing a normal respiratory rhythm before birth and in providing chemosensory drive.

\section{Introduction}

The retrotrapezoid nucleus (RTN) is a group of interneurons located near the medullary surface ventral to the facial nucleus (nVII). First identified as a site projecting extensively to the more caudal respiratory columns (Connelly et al., 1989; Smith et al., 1989), it has been attributed a role in central chemoreception, i.e., the activation of breathing by increased $\mathrm{pCO}_{2}$ through the detection of changes in $\mathrm{pH}$ (Nattie and Li, 2002; Mulkey et al., 2004; Abbott et al., 2009). RTN neurons are molecularly defined by expression of Vglut2, neurokinin1 receptor (NK1R), and the Phox $2 \mathrm{~b}$ transcription factor and lack of tyrosine hydroxylase (TH) (Stornetta et al., 2006; Onimaru et al., 2008). The $\mathrm{CO}_{2}-$

Received June 5, 2009; revised Sept. 22, 2009; accepted 0ct. 8, 2009.

This work was supported by grants from Agence nationale de la recherche (to G.F. and J.-F.B.), Fondation pour la Recherche Médicale (to J.-F.B.), and Association Française contre les Myopathies (to C.G.) and institutional support from Centre National de la Recherche Scientifique and Ecole normale supérieure. We thank Eva Coppola for the initial characterization of the $1 s$ let $1^{\text {cre }}$;Phox $2 b^{10 x / 10 x}$ mice and Carmen Le Moal for mouse husbandry. We thank Patrick Charnay for the Egr2 ${ }^{\text {cre }}$ (Krox20 $0^{\text {cre }}$ ) and Silvia Arber for the Islet ${ }^{\text {cre }}$ and Tau ${ }^{\text {MGFP }}$ mouse lines.

*V.D. and M.T.-B. contributed equally to this work.

Correspondence should be addressed to any of the following at the above addresses: Christo Goridis, E-mail: goridis@biologie.ens.fr; Gilles Fortin, E-mail: gilles.fortin@iaf.cnrs-gif.fr; or Jean-François Brunet, E-mail: jfbrunet@biologie.ens.fr.

K. Persson's present address: Department of Neuroscience and Pharmacology, The Panum Institute, 2200 Copenhagen N, Denmark.

M. Rallu's present address: Sanofi-Aventis R\&D, 94403 Vitry sur Seine Cedex, France.

A. Pattyn's present address: Institut des Neurosciences de Montpellier INSERM U583, 34091 Montpellier Cedex 5, France.

DOI:10.1523/JNEUROSCI.2623-09.2009

Copyright $\odot 2009$ Society for Neuroscience $\quad 0270-6474 / 09 / 2914836-11 \$ 15.00 / 0$ sensitive RTN neurons fire tonically, not rhythmically, in the adult rat (Nattie and Li, 2002; Mulkey et al., 2004). However in neonatal rats, the RTN overlaps anatomically with the parafacial respiratory group ( $\mathrm{pFRG}$ ) identified as a collection of intrinsically rhythmic preinspiratory neurons (Onimaru and Homma, 2003). The rhythmic pFRG neurons respond to $\mathrm{CO}_{2}$ and share marker gene expression with the RTN (Onimaru et al., 2008), suggesting that pFRG and RTN are the same group of cells that show tonic or rhythmic activities depending on experimental conditions or developmental stage. Respiratory network activity starts at embryonic day 15.5 (E15.5) in the mouse (ThobyBrisson et al., 2005). Moreover, a population of neurons termed $\mathrm{e}-\mathrm{pF}$ for embryonic parafacial neurons that display respiratorylike rhythmic activity are already detected at E14.5 in the parafacial region. They are intrinsically rhythmic, express NK1R and Phox2b (Thoby-Brisson et al., 2009), and as shown here, respond to a $\mathrm{pH}$ challenge, as do $\mathrm{pFRG}$ neurons in the newborn rat (Onimaru et al., 2008). They thus likely represent the embryonic forerunner of the pFRG.

A role for Phox 2b-expressing neurons in central chemosensitivity was first revealed by the observation that heterozygous mutations in $P H O X 2 B$ cause congenital central hypoventilation syndrome (CCHS) in man (Amiel et al., 2003; Weese-Mayer et al., 2005). Indeed, a cardinal symptom of CCHS is unresponsiveness to high $\mathrm{CO}_{2}$ (Spengler et al., 2001). Mice harboring the most frequent human PHOX2B mutation (the Phox $2 b^{27 A l a}$ allele) die at birth from respiratory failure, do not respond to hypercapnia, and have abnormal breathing patterns. The only anatomical de- 
fect found in these mutants is a massive depletion of RTN neurons, but functional deficits in other $P h o \times 2 b^{+}$structures cannot be excluded (Dubreuil et al., 2008). It is also unclear whether lack of central chemoreception is the sole cause of the fatal central apnea or whether the loss of rhythmogenic neurons in the parafacial region contributes to the lethal phenotype.

In this study, we set out to address these issues. We found that RTN neurons not only are sensitive to the toxic Phox $2 b^{27 A l a /+}$ mutation, but also require Phox $2 b$ for proper differentiation. This enabled us to generate two conditional Phox $2 b$ mutations, in which RTN development is disrupted. In hindbrain preparations of all three genetic conditions, the Phox $2 b^{27 A l a /+}$ and the two conditional Phox $2 b$ mutants, both the rhythmic activity and the response to a $\mathrm{pH}$ challenge were lost in the parafacial region. This suggests that, in addition to the loss of $\mathrm{CO}_{2} / \mathrm{pH}$ sensitivity, absence of a functional parafacial oscillator contributes to the lethal respiratory phenotype of the mutants.

\section{Materials and Methods}

Mice. To generate a floxed Phox $2 b\left(\right.$ Phox $\left.2 b^{\text {lox }}\right)$ allele, we flanked Phox $2 b$ exon 2, which contains the major part of the homeodomain, with loxP sites. The targeting vector, constructed by conventional cloning methods, contained from $5^{\prime}$ to $3^{\prime}$ (1) a 5' homology arm of $5 \mathrm{~kb},(2)$ a loxP site inserted into the first intron, (3) exon 2 and surrounding intron sequences, (4) a loxP site inserted into the second intron followed by a neomycin resistance cassette flanked by frt sites, and (5) a 3' homology arm of $4.5 \mathrm{~kb}$. The construct was electroporated into CK35 ES cells of $129 \mathrm{~S} v \mathrm{Pas}$ origin. Correctly targeted ES cells were expanded, confirmed by Southern blotting (supplemental Fig. 1, available at www.jneurosci. org as supplemental material) and injected into blastocysts (Service d'Expérimentation Animale et de Transgénèse) to generate chimeras that transmitted the mutant allele through the germ line. The agouti offspring of the chimeras were genotyped by PCR using the following primers flanking the $5^{\prime}$ loxP site: GGCCGGTCATTTTTATGATC and AAGTGCCTTTGGGTGAGATG.

The neomycin resistance cassette was removed by crossing with Flpe deleter mice (Rodríguez et al., 2000). The resulting heterozygous Phox $2 b^{\text {lox/+ }}$ mice were maintained on a mixed C57BL6/DBA (B6D2) genetic background for at least four generations and intercrossed to produce homozygous Phox $2 b^{\text {lox/lox }}$ mice, which are thus mainly B6D2 with some contribution of $129 \mathrm{SvPas}$. Phox $2 b^{\text {lox/lox }}$ mice were viable and fertile and without obvious phenotype. When crossed with Pgk::cre deleter mice (Lallemand et al., 1998), the resulting Phox $2 b^{\text {lox/lox }} ;$ Pgk:: cre embryos died at $\sim$ E13.5 as do the original Phox $2 b$-null mutants (Pattyn et al., 1999). Results to be published elsewhere (E. Coppola and J.-F. Brunet, unpublished results) show that introduction into the Phox $2 b^{\text {lox/lox }}$ background of the Brn4::cre allele expressed in all CNS progenitors (Zechner et al., 2003) resulted in a CNS phenotype identical to that seen in simple Phox $2 b$-null mutants (Pattyn et al., 2000). In Phox $2 b^{\text {lox/lox }}$ cells, in which the floxed Phox $2 b$ locus has been recombined, Phox $2 \mathrm{~b}$ protein is not detectable any more (Coppola and Brunet, unpublished results), thus providing a convenient readout of recombination efficiency. The generation of Phox $2 b^{27 A l a /+}$ (Dubreuil et al., 2008), Egr2 $2^{\text {cre/+ }}$ (Voiculescu et

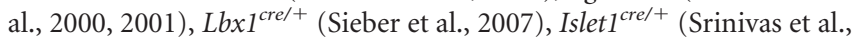
2001), and Tau ${ }^{m G F P}$ (Hippenmeyer et al., 2005) (called here Tau ${ }^{\text {GFPLAaC, }}$, since we used $L a c Z$ as reporter) mice has been described. Since heterozygous Phox $2 b^{27 A l a /+}$ mice die at birth, Phox $2 b^{27 A l a /+}$ embryos were produced by crossing male founder chimeras with B6D2 females (Dubreuil et al., 2008). In some experiments conditional Phox $2 b^{27 \text { Ala/+ }} ;$ Pgk::cre mutants to be described in detail elsewhere have been used. In this strain, the normal Phox $2 b$ exon 3 is replaced by an exon 3 bearing the Phox $2 b^{27 A l a}$ mutation in the presence of cre recombinase activity. While the mice bearing the conditional Phox $2 b^{27 A l a}$ allele are viable and fertile, crossing them with Pgk::cre deleter females results in a phenotype, which is undistinguishable from that of the previously described simple Phox $2 b^{27 \text { Ala } /+}$ mutants (data not shown). The Egr2 ${ }^{\text {cre/+ }}, \mathrm{Lbxl}^{\text {cre/+ }}$, Islet ${ }^{\text {cre/+ }}$, and Tau ${ }^{\text {GFPnLacZ }}$ lines were maintained on a B6D2 back- ground. Phox $2 b^{\text {lox/lox }}$ mice homozygous for the floxed Phox $2 b$ locus and harboring the Egr2 ${ }^{\text {cre }}, L b x 1^{\text {cre }}$, or Islet $1^{\text {cre }}$ alleles were produced by crossing Phox $2 \mathrm{~b}^{\text {lox/lox }}$ females with Phox $2 b^{\text {lox/+ }}$ males harboring the appropriate cre allele. In all cases, the day at which a vaginal plug was observed was considered as embryonic day 0.5 (E0.5). All animal studies were done in accordance with the guidelines issued by the French Ministry of Agriculture and have been approved by the Direction départementale des services vétérinaires de Paris.

Histology. The methods for in situ hybridization (ISH) coupled with immunohistochemistry and for double-fluorescence ISH on transverse and sagittal $12 \mu \mathrm{m}$ cryosections have been described (Tiveron et al., 1996; Hirsch et al., 1998; Dubreuil et al., 2008). Riboprobes for Atoh1 and Vglut2 (Slc17a6) were synthesized using a DIG RNA labeling kit (Roche) as specified by the manufacturer. The primary antibodies used were as follows: rabbit anti-Phox2b (1/2000) (Pattyn et al., 1997), guinea pig anti-Phox2b (1/600), rabbit anti-NK1R (1/1500) (Sigma), anti-tyrosine hydroxylase (1/500) (Millipore Bioscience Research Reagents), antiIslet 1,2 (1/100) (40.2D6 and 39.4D5, Developmental Studies Hybridoma Bank), and anti- $\beta$-galactosidase (1/1000) (Cappel or Abcam). The guinea pig anti-Phox $2 \mathrm{~b}$ antibody used in double-labeling experiments was produced against the same C-terminal peptide as the rabbit antibody (Pattyn et al., 1997). Its specificity was originally shown by the perfect match between the expression patterns given by the guinea pig and the rabbit antiserum and was confirmed by the absence of immunoreactivity in Phox $2 b$ knock-out mice. The primary antibodies were revealed for fluorescent staining by Alexa 488-, Cy3-, and Cy5-labeled secondary antibodies of the appropriate specificity (Jackson ImmunoResearch Laboratories) or for bright-field observation by biotin-labeled secondary antibodies and Vectastain ABC kit (Vector Laboratories) revealed with 3,3'-diaminobenzamide. Pictures were captured with either a Hamamatsu ORCA-ER or a Leica DFC420C camera mounted on a Leica DM5500B microscope for observation through fluorescence or bright-field optics, respectively, and then contrast-adjusted in Adobe Photoshop and assembled in Adobe Illustrator. RTN neurons were counted on transverse sections throughout an area delimited by the ventral borders of nVII and the medullary surface, starting with the rostralmost section containing nVII neurons and including a compact group of Phox $2 \mathrm{~b}^{+} ; \mathrm{TH}^{-}$;Islet $1,2^{-}$cells immediately caudal to nVII (Dubreuil et al., 2008). Triple-labeled Vglut2 ${ }^{+}$;

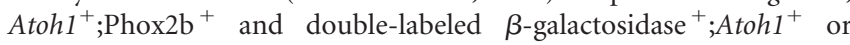
Phox $2 \mathrm{~b}^{+} ;$Atoh $1^{+}$cells were counted on every fourth section on both sides of the embryo.

In vitro preparations. Pregnant mice were killed by cervical dislocation between E14.5 and E16.5. Embryos were excised from the uterine horns and placed in oxygenated artificial CSF (a-CSF) at $20^{\circ} \mathrm{C}$ until the recording session. The a-CSF composition was as follows (in mM): $120 \mathrm{NaCl}, 8$ $\mathrm{KCl}, 1.26 \mathrm{CaCl}_{2}, 1.5 \mathrm{MgCl}_{2}, 21 \mathrm{NaHCO}_{3}, 0.58 \mathrm{Na}_{2} \mathrm{HPO}_{4}$, and 30 glucose, $\mathrm{pH} 7.4$, and was equilibrated with $95 \% \mathrm{O}_{2}-5 \% \mathrm{CO}_{2}$. Lowering the a-CSF $\mathrm{pH}$ to 7.2 was obtained by decreasing the $\mathrm{NaHCO}_{3}$ concentration to 10.5 $\mathrm{mm}$ and adjusting the $\mathrm{NaCl}$ concentration at $130.5 \mathrm{~mm}$. Brainstem and slice preparations were dissected in a-CSF at $4^{\circ} \mathrm{C}$. Brainstems were isolated from the embryo by a rostral section at the limit between the mesencephalon and the rhombencephalon and a section caudal to the fourth cervical root (C4). Transverse medullary slices isolating the preBötzinger complex (preBötC) were obtained as previously described (ThobyBrisson et al., 2005). Briefly, the brainstem embedded in an agar block was transversally sectioned by a vibratome from rostral to caudal until the posterior limit of nVII was reached. At a distance of $200 \mu \mathrm{m}$ caudal to it, a $450-\mu \mathrm{m}$-thick slice with the neuronal population of the preBötC being accessible at the anterior side of the slice was isolated. After isolation, preparations were incubated in Calcium Green-1 AM (see below) when appropriate and then transferred into a recording chamber and continuously perfused with oxygenated a-CSF at $30^{\circ} \mathrm{C}$. A 30 min recovery period was allowed before performing recordings. Brainstems were positioned ventral side up, and transverse slices were positioned rostral side up, and the resulting number was multiplied by 4 .

Calcium imaging. Preparations were incubated for $40 \mathrm{~min}$ in oxygenated a-CSF containing the cell-permeant calcium indicator dye Calcium Green-1 AM (10 $\mu \mathrm{M}$; Invitrogen). After loading, preparations were placed into the recording chamber, where the excess of dye was rinsed 
over a 30 min period. A standard epifluorescent illumination system on an E-600-FN upright microscope (Nikon) equipped with a fluorescein filter block was used to excite the dye and capture the emitted light. Fluorescence images were captured with a cooled CCD camera (Coolsnap HQ, Photometrics) using an exposure time of $100 \mathrm{~ms}$ in overlapping mode (simultaneous exposure and readout) during periods of $180 \mathrm{~s}$ and analyzed using MetaMorph software (Universal Imaging). The average intensity in regions of interest was calculated for each frame. Changes in fluorescence were expressed as a ratio of changes to the initial value $(\Delta F / F)$.

Electrophysiology. Recordings of cranial nerves in isolated brainstems and local population activity in transverse slices were performed using suction electrodes positioned at the proximal end of the facial nerve ( $7 \mathrm{n}$ ) or the $\mathrm{C} 4$ roots or on the surface of the slice in the preBötC region. The pipettes (tip diameter, 100-150 $\mu \mathrm{m}$ ) were filled with a-CSF, connected through silver wires to a high-gain AC amplifier (7P511; Grass Instrument). The collected signals were then filtered (bandwidth, $3 \mathrm{~Hz}$ to $3 \mathrm{kHz}$ ), integrated using an electronic filter (time constant $100 \mathrm{~ms}$, Neurolog system; Digitimer), recorded on a computer through a 1322A Digidata interface (Molecular Devices), and analyzed using PClamp9 software (Molecular Devices). Activity frequencies were measured over a 3 min period. Values are given as mean \pm SEM. Statistical significance was tested using a difference Student's $t$ test to compare frequencies obtained from different mutants, and a paired difference Student's $t$ test to compare the same preparation in two different conditions. The actual $p$ values are given for all statistically significant values $(p<0.01)$. Values of $p>0.1$ were considered as not significantly different from each other.

\section{Results}

\section{Developmental characterization of RTN neurons}

The term retrotrapezoid nucleus was coined by Smith et al. (1989) to name a group of neurons located in the parafacial region that could be retrogradely labeled from the dorsal and ventral respiratory groups. Despite its name, the rodent RTN is not a bona fide nucleus with a distinct architectonic appearance. Rather, RTN neurons are a sparse collection of small- to mediumsized neurons that occupy an area with ill defined borders, ventral and immediately lateral and caudal to nVII (Cream et al., 2002; Takakura et al., 2008). Therefore, RTN neurons are best defined at this time by their functional and molecular properties: they are $\mathrm{CO}_{2}$-responsive, glutamatergic $\left(\right.$ Vglut $\left.^{+}\right)$, TH-negative, Phox2bexpressing neurons, located parafacially near the medullary surface, that also express NK1R (Nattie and Li, 2002; Stornetta et al., 2006; Dubreuil et al., 2008; Onimaru et al., 2008; Pagliardini et al., 2008; Takakura et al., 2008). This definition leaves out probably only a minority of neurons in the same location since $>90 \%$ of the neurons under the lateral two-thirds of nVII appear to express Phox $2 \mathrm{~b}$ in the adult rat (Takakura et al., 2008). Also in neonatal rats, $82 \%$ of preinspiratory and virtually all of the chemosensitive pFRG neurons were reported to be Phox $2 \mathrm{~b}^{+}$(Onimaru et al., 2008). Moreover, chemosensitivity, expression of Phox $2 b$, Vglut2, and NK1R, negativity for TH and location are diagnostic criteria to identify these neurons in fetal (this paper), newborn (Onimaru et al., 2008), and adult (Stornetta et al., 2006) rodents. We will thus use the term RTN neurons to refer to the neurons located ventral and immediately lateral and caudal to nVII that are Phox $2 \mathrm{~b}^{+} ;$Vglut $^{+}, \mathrm{NK}_{1 \mathrm{R}}{ }^{+}$, and TH-negative, adding the criterion of Atohl expression (see below). Their precise electrophysiological properties, however, appear to change between fetal, neonatal, and adult stages (Mulkey et al., 2004; Stornetta et al., 2006; Onimaru et al., 2008; Thoby-Brisson et al., 2009), and we will use the terms e-pF or pFRG when referring explicitly to the electrophysiological properties of these neurons in fetal and newborn rodents.

RTN neurons share the molecular signature Phox $2 \mathrm{~b}^{+}$; $\operatorname{Vglut}^{+} ; \mathrm{NK} 1 \mathrm{R}^{+}$with other interneurons in the medulla and can thus not be distinguished before they settle in their final location at $\sim$ E14. We therefore searched for additional markers, and found that the great majority (85\%, range 77-93 for 34 sections from two embryos) of the Phox $2 b^{+} ;$Vglut $2^{+}$neurons in the RTN expressed the transcription factor Atoh1 (Math1) in late gestation. Conversely, virtually all (98-99\%) Atoh $1^{+}$cells in the region coexpressed Phox $2 b$ and Vglut2 (Fig. 1A-D). Also at E15.5, all NK1R ${ }^{+}$RTN neurons that could be clearly identified coexpressed Atoh1 (Fig. 1 E-G) (since NK1R ${ }^{+}$cell bodies are difficult to identify because of the predominance of stained processes, we did not attempt to quantify the proportion that also expresses Atoh1). There was no overlap between TH and Atoh1 expression (Fig. $1 H-J)$. Some $\left(<15 \%\right.$ of all Atoh $1^{+}$cells in the ventral medulla at E14.5) Atoh ${ }^{+} ;$Phox $2 \mathrm{~b}^{+}$cells were found dorsal to nVII (supplemental Fig. 2, available at www.jneurosci.org as supplemental material). They expressed Vglut2 and were derived from $\mathrm{Lbx1}^{+}$and $\mathrm{Egr}^{+}$precursors (data not shown) like bona fide RTN neurons (see below). Their molecular phenotype and origin thus resembles that of RTN neurons. One may speculate that RTN neurons are part of a greater ensemble of perifacial neurons with partially similar functions. We did not observe other Atoh $1^{+}$cells in the medulla except for ventricular zone cells in the rhombic lip (Fig. $1 K-N$ ), a known Atoh1-expressing site (Akazawa et al., 1995; Wang et al., 2005), and a stream of cells at its lateral edge (Fig. $1 \mathrm{~N}$ ) that may correspond to the forming cochlear nucleus, a rhombic lip derivative (Wang et al., 2005; Fujiyama et al., 2009). In the ventral medulla, Atoh1 thus is a specific marker of RTN neurons defined as cells expressing Phox2b, Vglut2, and NK1R but not TH. A schematic of the rostrocaudal distribution of Atoh $1^{+}$cells in the medulla is represented in Figure $1 O$.

The specificity of Atoh1 expression for RTN neurons opened the way to trace RTN precursors at earlier stages of development. At E13.5, a facial nucleus has already formed while some nVII precursors are still migrating (Fig. $1 \mathrm{M}$ ). At this stage, cells coexpressing Phox $2 \mathrm{~b}$ and Atoh 1 have arrived at the medullary surface at the lateral edge of nVII. One day earlier, Atoh $1^{+} ; \mathrm{Phox} 2 \mathrm{~b}^{+}$cells formed a stream of cells just lateral to the migrating $\mathrm{nVII}$ precursors (Fig. $1 \mathrm{~L}$ ). No Atoh $1^{+}$cells were ever observed in the gap between the Atoh $1^{+}$;Phox $2 \mathrm{~b}^{+}$stream of cells and the Atoh $1^{+}$ rhombic lip. This together with the fact that the rhombic lip and its derivatives are Phox $2 b$-negative makes it unlikely that the Atoh 1;Phox $2 \mathrm{~b}$ double-positive cells originate from this structure. The impression of a greater number of $A$ toh $1^{+} ;$Phox $2 \mathrm{~b}^{+}$neurons at E13.5 than at later stages is caused by the lesser dispersal of the cells that do not yet extend caudal to nVII (data not shown). At E11.5, cells coexpressing Atoh1 and Phox2b could be observed neither in rhombomere 4 (r4), where the nVII neurons arise, nor in the adjacent rhombomeres (Fig. $1 \mathrm{~K}$ and data not shown). These results suggest that Atoh1 expression is switched on between E11.5 and E12.5 in RTN precursors, which join the facial motor neurons in their migration toward the pial surface of $\mathrm{r} 6$.

The conspicuous comigration of the Atoh $1^{+} ; \mathrm{Phox} 2 \mathrm{~b}{ }^{+} \mathrm{RTN}$ precursors with that of nVII prompted us to ask whether the latter were required for migration of the former to the ventral medulla. To test this idea, we made use of conditional Phox $2 b$ $\left(P h o x 2 b^{\text {lox/lox }}\right)$ mutants that express cre recombinase from the Islet 1 locus in all motoneuron precursors (Srinivas et al., 2001). In Phox $2 b^{\text {lox/lox }}$; Islet $1^{\text {cre/+ }}$ embryos, the development of facial neuron precursors is compromised, and they do not migrate into r6 (Coppola and Brunet, unpublished results). This did not prevent the Atoh $1^{+}$cells from migrating toward the ventral pial surface of r6, although they remained somewhat more dispersed and were 

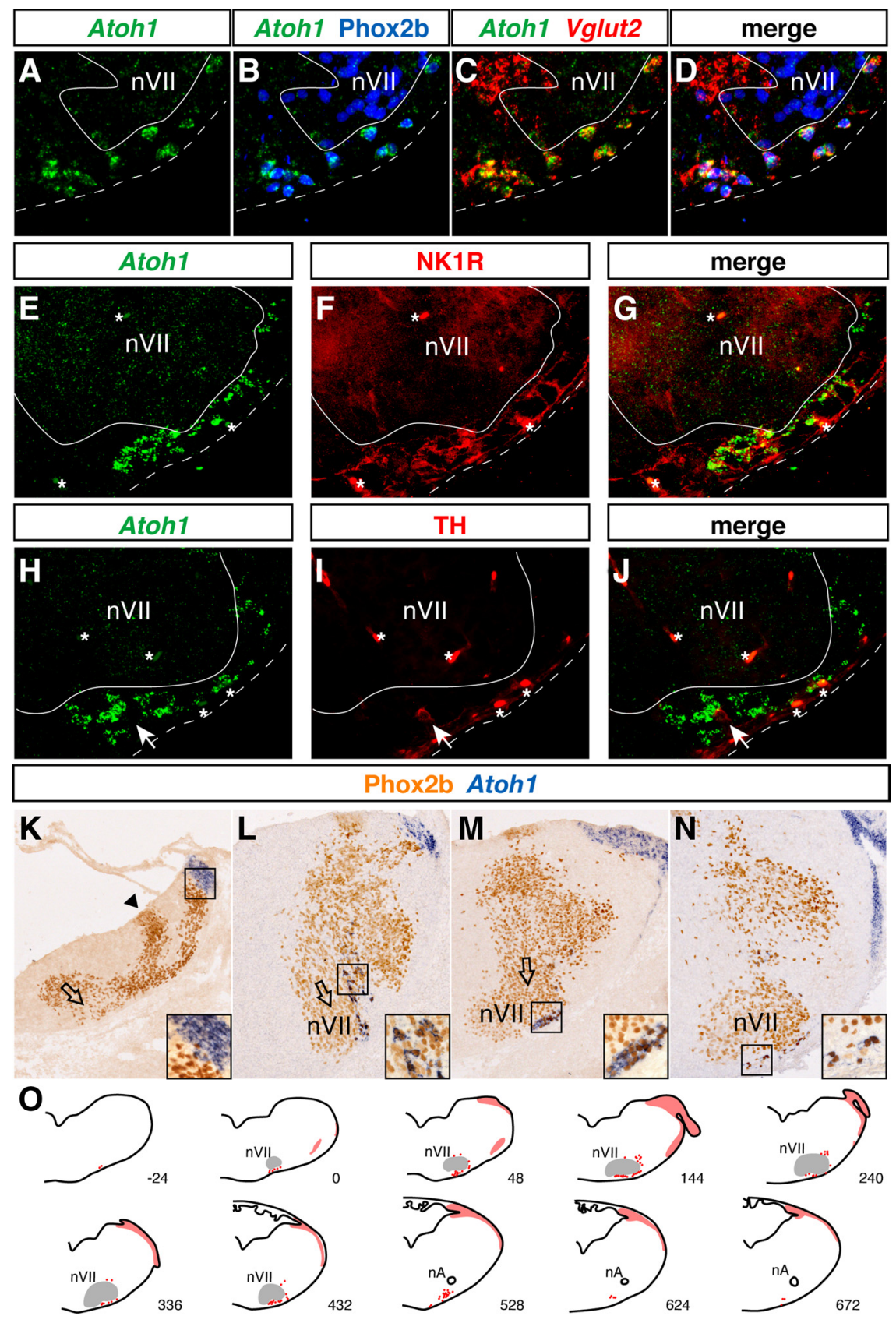

Figure 1. Characterization of Atoh1-expressing neurons in the RTN. $\boldsymbol{A}-\boldsymbol{J}$, Transverse sections through E18.5 (A-D) and E15.5 $(\boldsymbol{E}-\boldsymbol{J})$ medulla. $\boldsymbol{A}-\boldsymbol{D}$, ISHs with Atoh1 ( $\boldsymbol{A}$, green) and Vglut2 ( $\boldsymbol{C}$, red) probes combined with anti-Phox2b immunofluorescence ( $\boldsymbol{B}$, blue). All Atoh1-positive cells express Phox $2 \mathrm{~b}$ and $V$ glut2. The sections shown in $\boldsymbol{A}-\boldsymbol{D}$ are through the rostral end, those shown in $\boldsymbol{E}-\boldsymbol{J}$ through the center of nVII. $\boldsymbol{E}-\boldsymbol{J}$, ISH with an Atoh1 probe ( $\boldsymbol{E}$ and $\boldsymbol{H}$, green) followed by anti-NK1R $(\boldsymbol{F})$ or anti-TH $(\boldsymbol{I})$ immunofluorescence (red). Atoh1-positive cells express NK1R (G) but not TH $(\boldsymbol{J})$. A white arrow points to a $\mathrm{TH}^{+}$; Atoh $^{-}$cell. A white line delimits nVII, and a dotted line marks the ventral medullary surface; lateral is to the right. Asterisks, Nonspecific signal from blood vessels. $\boldsymbol{K}-\boldsymbol{N}$, Transverse sections through r6 at E11.5 (K), E12.5 $(\boldsymbol{L})$, E13.5 $(\boldsymbol{M})$, and E15.5 $(\boldsymbol{N})$ labeled by Atoh1 ISH (blue) and anti-Phox2b immunohistochemistry (orange). The insets show enlargements of the framed regions. Note the absence of double-positive cells at E11.5 $(\boldsymbol{K})$. A black arrowhead points to the Phox $2 b^{+} \mathrm{dB} 2$ progenitor domain $(\boldsymbol{K})$. Open arrows indicate the $n V I I$ precursor migratory stream to the pial surface of $r 6.0$, Schematic representation of Atoh 1 expression in transverse sections through the medulla of an E14.5 embryo. The red dots represent the parafacially located Atoh $1^{+}$cells in the ventral medulla, the red areas the strongly Atoh $1^{+}$cells of the rhombic lip and its derivatives. The distance from the rostral end of $n V I I$ is given in $\mu \mathrm{m}$ below each section. nA, Nucleus ambiguus.

less clustered near the medullary surface than in wild-type embryos (Fig. 2A-D). This result also shows that the Atoh $1^{+}$RTN neurons retain Phox $2 \mathrm{~b}$ expression and thus do not derive from Islet1-expressing motoneuron precursors (Varela-Echavarría et al., 1996; Pattyn et al., 2000).
No Atoh $^{+}$cells were detectable in the ventral medulla of Phox $2 b^{27 \text { Ala/+ }}$ embryos, in which RTN neurons are severely depleted (Dubreuil et al., 2008), neither at E15.5 nor at E12.5, when Atoh1 expression first appears (Fig. 2E-H). Hence, RTN precursors are already affected by the mutation at this early stage. The almost complete loss of Atoh1-expressing cells in the RTN region is more severe than that of the cells defined by Phox $2 b$, Vglut2, and NK1R expression. This raises the possibility that the Atoh $1^{+}$cells represent a major but distinct subpopulation of the RTN neurons as defined here.

\section{RTN development depends on Phox $2 b$}

Although the RTN neurons are depleted in the Phox $2 b^{27 A l a /+}$ mutants, this does not mean that they depend on Phox $2 b$ for proper development. Rather, the selective demise of these neurons is likely caused by a toxic gain-of-function effect. It is thus not known what happens to them in the absence of Phox 2 b. In Phox $2 b$-null mutants, the status of the RTN is difficult to assess because of the absence of nVII (Pattyn et al., 2000), a key topological criterion to identify these neurons. We therefore sought to test the Phox $2 b$ dependence of the RTN by a conditional knockout strategy.

By genetic tracing, partnering either the $L b \times 1^{\text {cre }}$ or the Egr2 $2^{\text {cre }}$ allele with the Tau ${ }^{\text {GFPLAaZ }}$ reporter, we first confirmed published evidence (Pagliardini et al., 2008; Thoby-Brisson et al., 2009) that the majority of RTN neurons arise from the $L b \times 1^{c r e}$-expressing domain of the alar plate (Sieber et al., 2007), in the Egr2 ${ }^{\text {cre }}$ expressing r3 or r5 (Voiculescu et al., 2001) (Fig. 3A-J). A minor subpopulation of the Phox $2 \mathrm{~b}^{+} ;$Islet $1,2^{-} ; \mathrm{TH}^{-}$cells did not express the lineage marker in $L b \times 1^{\text {cre }}$;Tau ${ }^{\text {GFPnLacZ }}$ embryos, either because they do not derive from $L b x 1^{+}$precursors or because recombination of the Tau ${ }^{\text {GFPnLacZ }}$ locus is incomplete. In addition to RTN neurons, nearby $\mathrm{TH}^{+}$; Phox $2 \mathrm{~b}^{+} \mathrm{C} 1$ neurons (Tiveron et al., 1996; Takakura et al., 2008) also expressed the lineage tracer in Egr2 ${ }^{\text {cre }} ;$ Tau $^{\text {GFPnLacZ }}$ (but not in $L b x 1^{\text {cre }}$; Tau ${ }^{\text {GFPnLacZ }}$ ) embryos (Fig. $3 F-I$ ), indicating that these cells derive from $\mathrm{r} 3 / 5$ as well. We extended these results using double labeling for Atoh 1 and $\beta$-galactosidase (Fig. $3 E, J$ ). In the RTN region, most Atoh ${ }^{+}$cells in both $\mathrm{Egr2}^{\text {cre }}$; Tau ${ }^{\text {GFPnLacZ }}$ (86.7\%, range 86.5-86.9, for 42 sections from 2 embryos) and $L b \times 1^{c r e} ; T_{a u}^{G F P n}$. LacZ (80.6\%, range 79.6-81.6, for 36 sections from 2 embryos) mutants also expressed the lineage marker. These results rule out that the $A$ toh $1^{+}$cells in the ventral medulla are derived from the 
rhombic lip, whose progeny does not express Lbxl at any stage (Sieber et al., 2007).

To investigate the Phox $2 b$ dependence of the RTN, we thus relied on Phox $2 b^{\text {lox/lox }}$ mice, in which the floxed second exon of Phox $2 b$ is removed by cre recombinase expressed from either the $L b x 1$ or the Egr2 locus. In these conditional mutants, nVII is preserved thus greatly facilitating the identification of the RTN. Similar to what has been observed in the Phox $2 b^{27 \text { Ala/ }+}$ mutants (Dubreuil et al., 2008), in Phox $2 b^{\text {lox/lox }} ; L b \times 1^{\text {cre/+ }}$ embryos, the NK1R immunoreactivity normally concentrated at the medullary surface in the parafacial area was almost completely lost (Fig. $4 A, B)$. We used Atoh 1 expression as additional criterion. Atoh1 expression had disappeared in E15.5 Phox $2 b^{\text {lox/lox }} ; L b \times 1^{\text {cre/+ }}$ mutants (Fig. 4C,D). The same result was obtained with Phox $2 b^{\text {lox/lox }} ; \mathrm{Egr}^{\text {cre/+ }} \mathrm{em-}$ bryos (Fig. $4 E, F$ ). In serial sections through the RTN region from both mutants, $4-12$ Atoh $1^{+}$cells were still detected compared with 1176 (range 1024-1328) cells in the controls $(n=3)$. To exclude adverse effects caused by the sole expression of cre recombinase (Naiche and Papaioannou, 2007), we used as controls animals expressing $L b x 1^{\text {cre }}$ or Egr ${ }^{\text {cre }}$ in some experiments. There were no noticeable effects on RTN development (data not shown and Fig. $4 A$ ).

Together, these results show that RTN neurons require Phox $2 b$ activity for proper differentiation and that conditional ablation of Phox $2 \mathrm{~b}$ in either Phox $2 b^{\text {lox/lox }} ; L b x 1^{\text {cre/+ }}$ or Phox $2 b^{\text {lox/lox }}$; $E g r 2^{\text {cre/+ }}$ mice can be used to impair RTN development. Since $L b \times 1$ is switched on only once the neurons have become postmitotic (Sieber et al., 2007), the results also show that Phox $2 b$ is required postmitotically in RTN neurons.

\section{Perinatal lethality of conditional Phox $2 b$ mutants}

We then explored the functional consequences of RTN ablation by conditional inactivation of Phox $2 b$ with either the $L b \times 1^{\text {cre }}$ or the Egr $2^{\text {cre }}$ allele. Conditional knock-outs $\left(P h o x 2 b^{\text {lox } / l o x} ; E g r 2^{\text {cre/+ }}\right.$ and Phox $2 b^{\text {lox/lox }} ; L b x 1^{\text {cre/+ }}$, respectively) were recovered in Mendelian proportions at E18.5. However, 1 week after birth, no Phox $2 b^{\text {lox/lox }} ; \mathrm{Egr}^{\text {cre/+}}$ pup (out of a total of 21 offspring) and only 4 Phox $2 b^{\text {lox/lox }} ; L b \times 1^{\text {cre/+ }}$ pups (out of a total of 48 offspring) were found alive instead of the expected $25 \%$, indicating that most had died in the neonatal period. The escapers of genotype Phox $2 b^{\text {lox/lox }} ; L b \times 1^{\text {cre/+ }}$ were smaller than their littermates but survived once past the critical newborn period. The less severe phenotype of the latter may be caused by the later (postmitotic) stage of recombination allowing some degree of RTN development or by differences between strains.

\section{The embryonic parafacial oscillator does not form in \\ Phox $2 b$ mutant embryos}

RTN neurons are massively depleted in Phox $2 b^{27 A l a /+}$ mice and, as shown here, in conditional Phox $2 b$ mutants. Consistent with a major role of the RTN in central chemosensitivity, the Phox $2 b^{27 \text { Ala/+ }}$ mutants completely lack $\mathrm{CO}_{2}$ responsiveness at birth (Dubreuil et al., 2008). However, participation in the central chemoreflex (i.e., the activation of breathing by elevation of

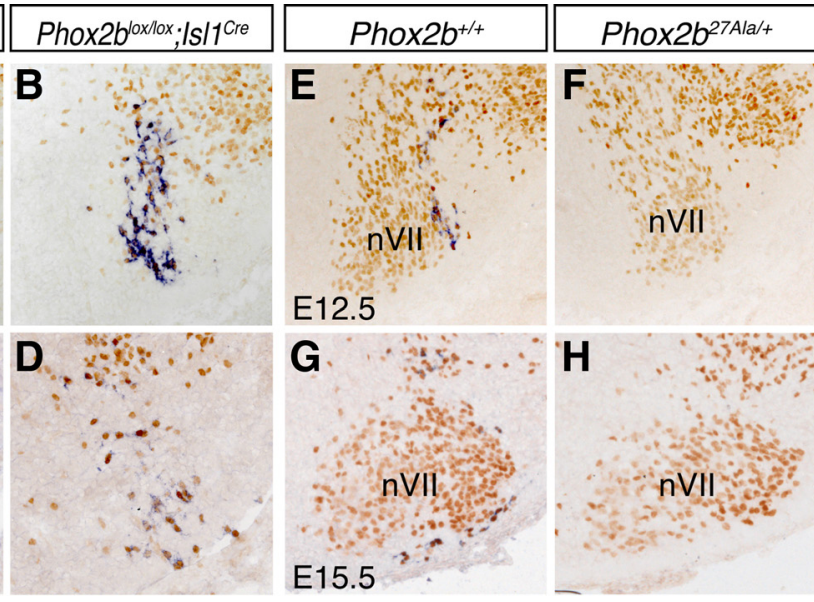

Figure 2. The Atoh1-expressing RTN neurons are preserved in $I s l e t 1^{\text {cre/+}}$ Phox2b conditional null mutants and lost in Phox $2 b^{27 A l a /+}$ mutants. $\boldsymbol{A}-\boldsymbol{H}$, ISH with an Atoh1 probe (blue) followed by anti-Phox2b immunohistochemistry (orange) on slet $^{\text {cre/+ }}(\boldsymbol{B}, \boldsymbol{D})$ embryos and through E12.5 $(\boldsymbol{E}, \boldsymbol{F})$ and E15.5 $(\boldsymbol{G}, \boldsymbol{H})$ brains of wild-type $(\boldsymbol{E}, \boldsymbol{G})$ and Phox $2 b^{27 A l a /+}(\boldsymbol{F}, \boldsymbol{H})$ brains. The ventral medullary surface is down, and lateral is to the right.

$\mathrm{pCO}_{2}$ through the detection of interstitial fluid acidification) may be only one aspect of the role of the RTN in breathing. In newborn rats, the parafacial region harbors rhythmically active neurons that are also $\mathrm{CO}_{2}$ sensitive and share with RTN neurons expression of Vglut2, NK1R, and Phox2b (Onimaru et al., 2008). In addition, a population of spontaneously rhythmic neurons termed e-pF and expressing Phox $2 \mathrm{~b}$ and NK1R is present in the same area already at E14.5. This population most likely represents the embryonic form of the pFRG (Thoby-Brisson et al., 2009). We therefore investigated whether Phox $2 b$ mutant embryos lacked rhythmic activity in the parafacial region.

By optical recording of brainstem en bloc preparations loaded with Calcium Green-1 AM, we identified the e-pF oscillator in control E14.5 embryos as a parafacially located rhythmic cell population (bursting frequency $11.1 \pm 1.1$ bursts/min) (Fig. $5 A$ ), in line with our previous results (Thoby-Brisson et al., 2009). In contrast, there was a complete absence of rhythmic fluorescence changes in the parafacial area of Phox $2 b^{27 A l a /+}$, Phox $2 b^{l o x / l o x}$; $L b \times 1^{\text {cre/+ }}$, and Phox $2 b^{\text {lox/lox }} ; E g r 2^{\text {cre/+ }}$ littermates (Fig. 5B-D). In these experiments, we used Phox $2 b^{+/+}$or Phox $2 b^{\text {lox/+ }}$ embryos as controls; our previous results have shown that the mere presence of cre recombinase expressed from the Egr2 locus was without effect (Thoby-Brisson et al., 2009). Since we did not observe differences between Phox $2 b^{+/+}$and Phox $2 b^{l o x /+}$ embryos, we pooled the data for the two genotypes. In control embryos, approximately half of the e-pF oscillations were accompanied by fluorescence changes in $\mathrm{nVII}$ and by concomitant bursts of activity recorded from the facial nerve root (7n) (Fig. 5A). A rhythmic motor activity was thus already present, but the coupling with the e-pF oscillations was still imperfect at this stage (Thoby-Brisson et al., 2009). In the mutants, occasional bursts of motor activity could be recorded, but their frequency was substantially reduced $\left(2.7 \pm 0.4\right.$ bursts $/ \mathrm{min}$ for Phox $2 b^{27 \mathrm{Ala} /+}, 2.6 \pm 0.2 \mathrm{bursts} / \mathrm{min}$ for Phox $2 b^{\text {lox/lox }} ; E g r 2^{\text {cre } /+}$, and $1.7 \pm 0.2$ bursts/min for Phox $2 b^{\text {lox/lox }}$; $L b \times 1^{\text {cre/+ }}$ embryos) (Fig. 5B-D). A low $\mathrm{pH}$ challenge markedly accelerated the rhythm of both the e-pF oscillations and the motor bursts in control embryos (Fig. 5A), but had no effect in the three types of mutants (Fig. $5 B-D$ ).

Together these results show first, that the responsiveness to $\mathrm{CO}_{2} / \mathrm{pH}$ demonstrated in the rhythmic $\mathrm{pFRG}$ neurons from neo- 


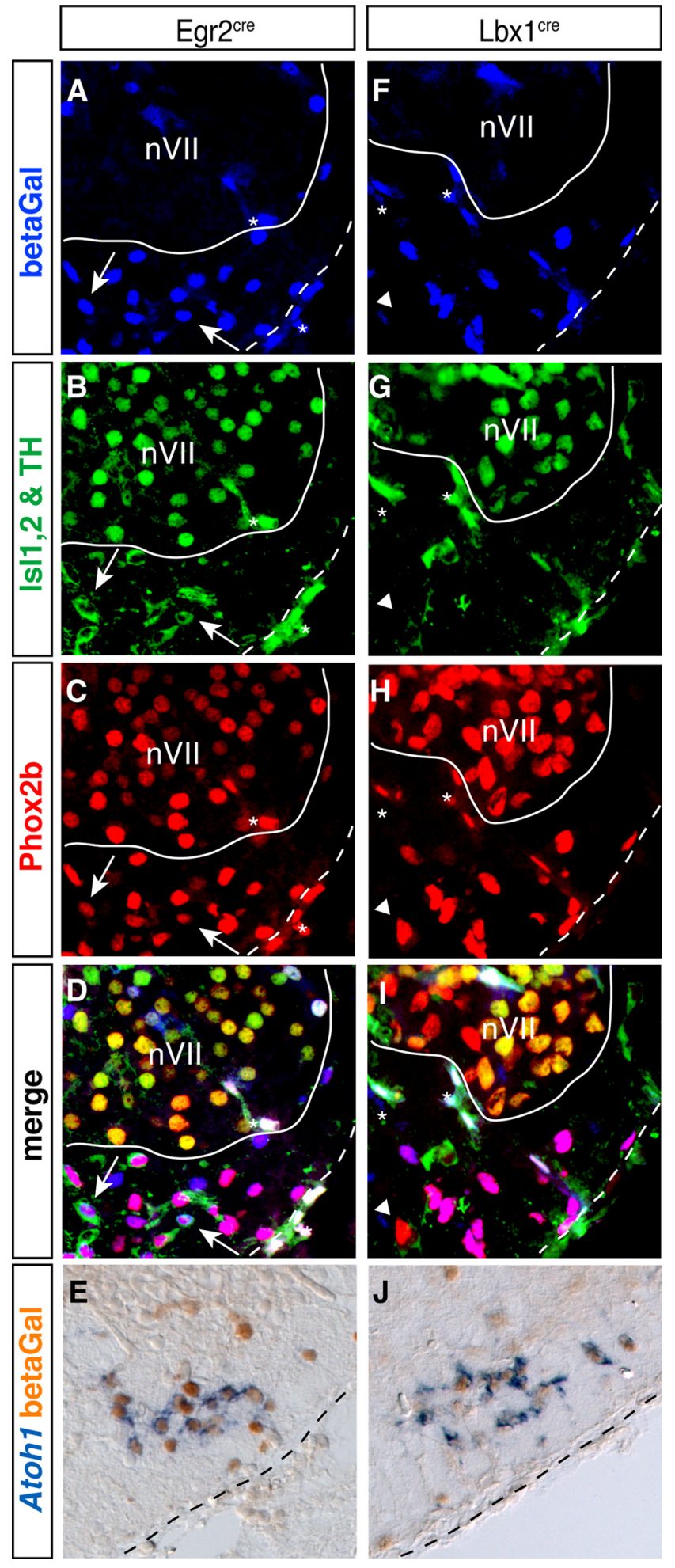

Figure 3. RTN neurons originate from Lbx1- and Egr2-expressing precursors. $A-J$, Trans-

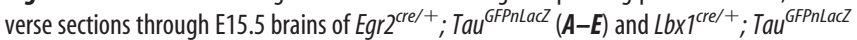
$(\boldsymbol{F}-\boldsymbol{J})$ embryos. $\boldsymbol{A}-\boldsymbol{D}, \boldsymbol{F}-\boldsymbol{I}$, Anti- $\beta$-galactosidase (blue, $\boldsymbol{A}$ and $\boldsymbol{F}$ ), combined anti-Islet1,2 (nuclear labeling) and anti-TH (cytoplasmic labeling) (green, $\boldsymbol{B}$ and $\boldsymbol{G}$ ), and anti-Phox2b (red, $\boldsymbol{C}$ and $\boldsymbol{H})$ immunofluorescence. A white line delimits nVII, and a dotted line marks the ventral med-

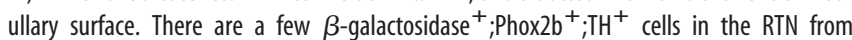
$\mathrm{Egra}^{\text {cre/+}}$; Tau ${ }^{\text {GFPnLaCZ }}$ embryos (white arrows) and one Phox $2 \mathrm{~b}^{+} ; \mathrm{TH}^{-}$;islet $1 / 2^{-}$cell that is

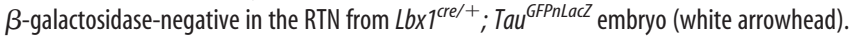
Asterisks, nonspecific signal from blood vessels. $E, J$, ISH with an Atoh 1 probe (blue) followed by anti- $\beta$-galactosidase immunohistochemistry (orange). A black dotted line marks the ventral medullary surface, lateral is to the right.
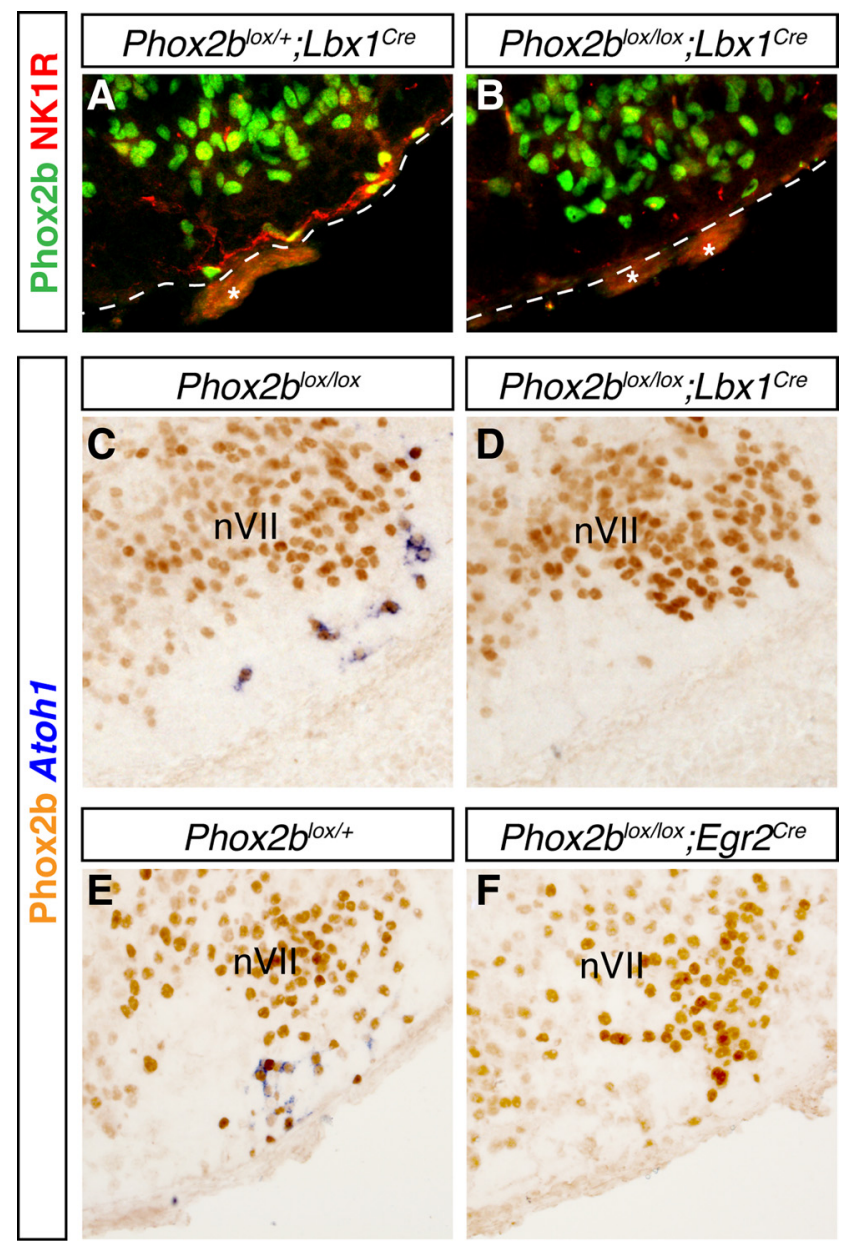

Figure 4. Loss of RTN neurons in Phox2b conditional null mutants. $A, B$, anti-Phox $2 b$ (green) and anti-NK1R (red) immunofluorescence on transverse sections through E15.5 brains of

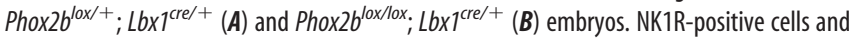
fibers at the ventral medullary surface beneath $\mathrm{nVII}$ are absent in the mutant embryo $(\boldsymbol{B})$. A dotted line marks the ventral medullary surface. Asterisks, Nonspecific signal from blood vessels. C-F, ISH with an Atoh 1 probe (blue) and anti-Phox2b immunohistochemistry (orange) on

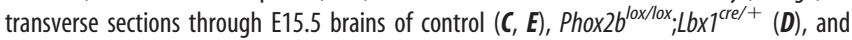
Phox $2 b^{\text {lox/lox; }}$ Egr2 ${ }^{\text {cre/+ }}(\boldsymbol{F})$ conditional mutants. Note the absence of Atoh1 staining in the conditional null mutants $(\boldsymbol{D}, \boldsymbol{F})$.

natal rats is already found in its embryonic forerunner, the e-pF, as soon as it becomes active at E14.5. Second, they show that in mice bearing the Phox $2 b^{27 A l a}$ or the conditional Phox $2 b$ mutations, the e-pF, which entrains the preBötC oscillator and increases the frequency of its oscillations (Thoby-Brisson et al., 2009), is not active. Third, in contrast to what is seen in the controls, the e-pF and the motor outflow are insensitive to acidification in the Phox $2 b$ mutants. Hence, three different Phox $2 b$ mutants in which RTN neurons are depleted show defective rhythm generation and chemosensitivity in the parafacial area.

\section{Emergence of the preBötzinger complex in}

Phox $2 b^{27 \text { Ala/+ }}$ mutants

The fact that preBötC neurons do not express Phox $2 b$ and should thus not be affected directly by the Phox $2 b^{27 A l a}$ mutation prompted us to test the status of the preBötC in the Phox $2 b^{27 A l a /+}$ mutant embryos. We thus recorded the neuronal activity of the preBötC in transverse medullary slices from E15.5 Phox2 $b^{27 A l a /+}$ and wild-type littermates (Fig. 6A,B). Extracellular recording of electrical activity and imaging of calcium transients revealed the 
A Phox $2 b^{+/+}$
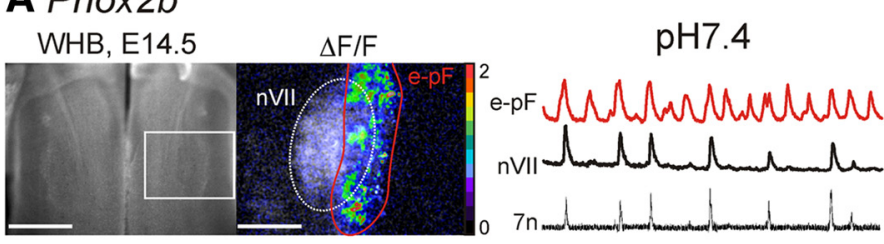

B Phox $2 b^{27 \text { Ala/t+ }}$
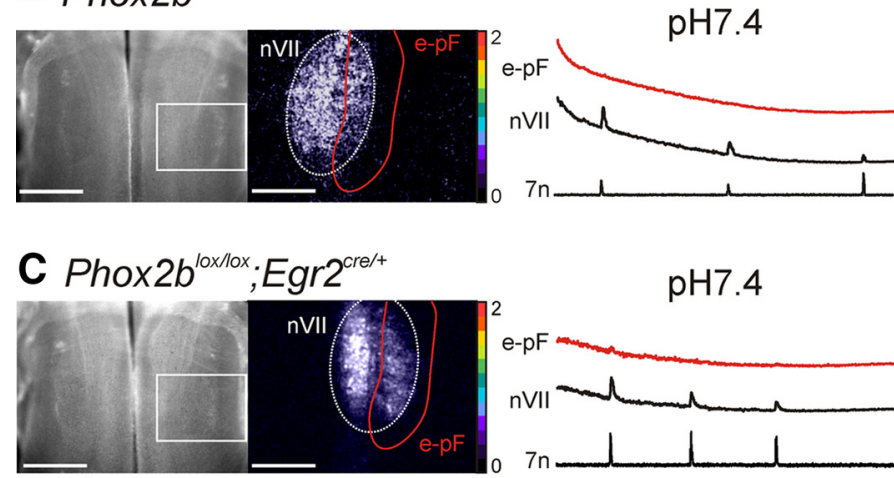

\section{Phox $2 b^{10 \times 1 / 0 x} ; L b \times 1^{\text {crelt }}$}

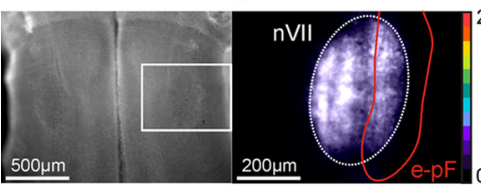

$\mathrm{pH} 7.4$

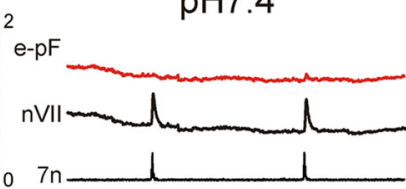

$\mathrm{pH} 7.2$

$\Delta F / F 2 \%$
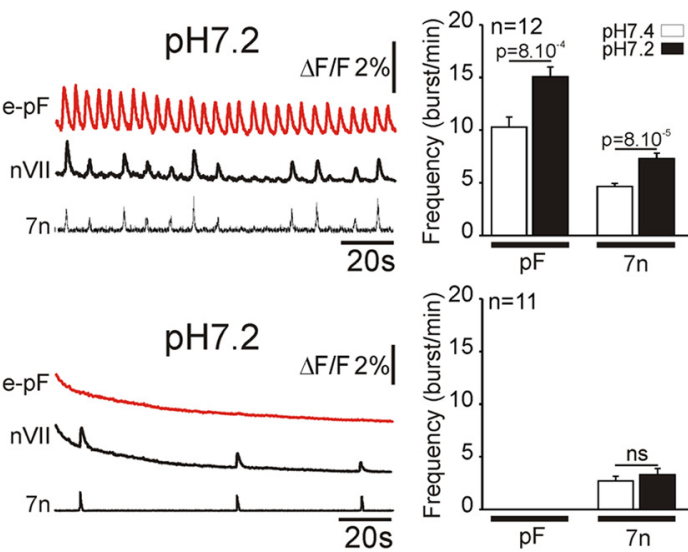

$\mathrm{pH} 7.2$
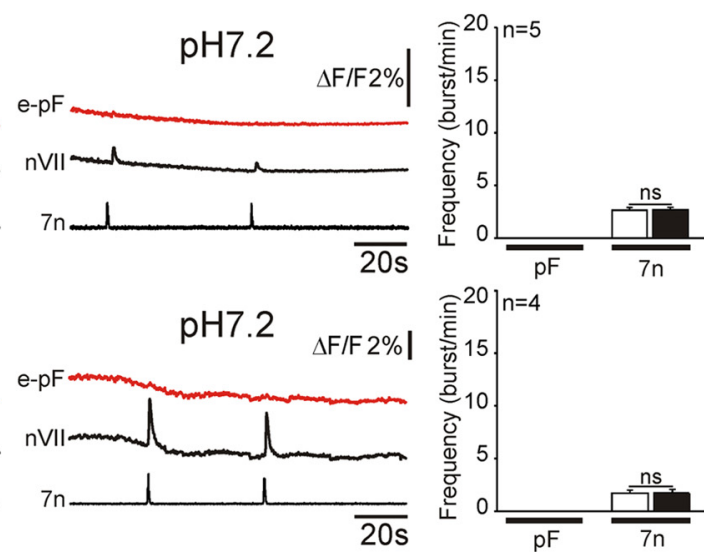

Figure 5. Loss of rhythmic activity in the parafacial area of brainstem preparations from Phox2b mutant embryos. Left panels, Photomicrographs of whole-hindbrain (WHB) preparations from E14.5 mouse embryos loaded with Calcium Green-1 AM (left) and images of spontaneous calcium transients recorded in the facial area (delimited by the rectangle in the leftmost panel) are shown (right). One image taken during a facial burst of activity (appearing in white on the $\Delta F / F$ panel) was used to position the nVII outlined by a white line. The calcium transients integrated over the e-pF/RTN area outlined in red are shown as relative changes in fluorescence $(\Delta F / F)$. Middle panels, Tracings of the calcium transients integrated over the e-pF/RTN area (outlined in red in the left panels) and of the rhythmic motor activity recorded from $\mathrm{nVII}$ and the root of the facial nerve $(7 \mathrm{n})$ at pH 7.4 and pH7.2. Right panels, Quantification of the frequency of rhythmic bursts of the e-pF (pF) and $7 \mathrm{n}$ as indicated. $p$ values are $8 \times 10^{-4}$ and $8 \times 10^{-5}$ for the differences in e-pF and $7 \mathrm{n}$ burst frequencies, respectively, between the two $\mathrm{pH}$ conditions in the control embryos. $\mathrm{ns}, p>0.1 ; n$, number of hindbrain preparations analyzed. $A$, Calcium transients and motor

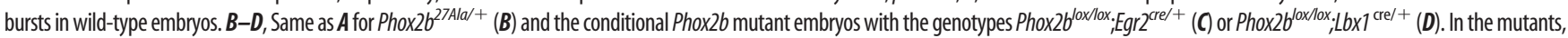
there is a complete absence of rhythmic activity in the e-pF/RTN that is not restored by low $\mathrm{pH}$ and a markedly reduced frequency of motor bursts that is equally $\mathrm{pH}$ unresponsive.

A

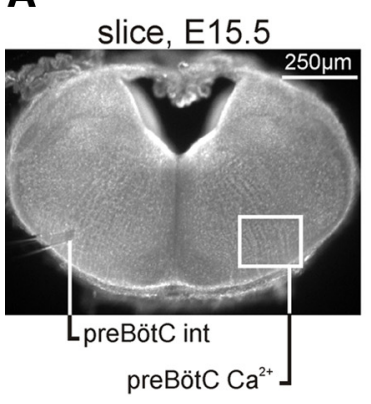

B Phox $2 b^{\text {t+ }}$

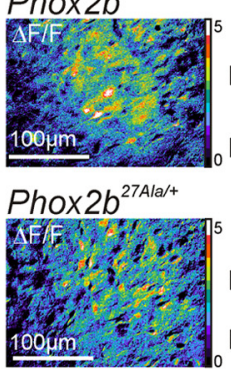

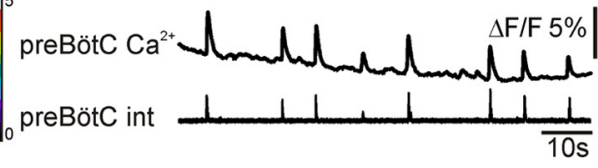

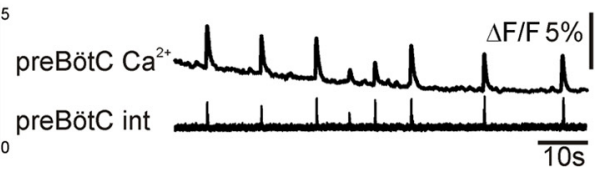

C

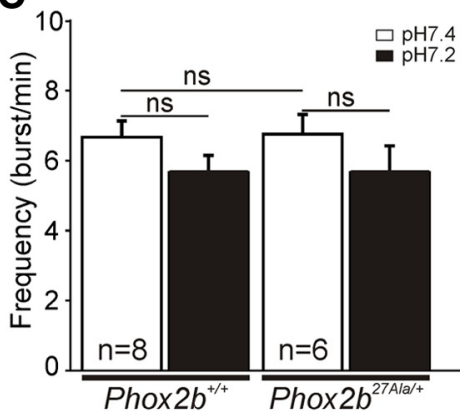

Figure 6. Emergence of a functional preBötC in Phox $2 b^{27 A l a}$ embryos. A, Photomicrograph of an E15.5 transverse medullary slice through the preBötC loaded with Calcium Green-1 AM. The positioning of the electrode for population recording from the preBötC (preBötC int) and the area (preBöt $\mathrm{Ca}^{2+}$ ) in which spontaneous calcium transients were measured are indicated. $B$, Combined calcium imaging and recording of population activity in Phox $2 b^{+/+}$and Phox $2 b^{27 A / a /+}$ embryos as indicated. Shown are images of the relative changes in fluorescence $(\Delta F / F)$ and the calcium transients recorded over the preBötC area (upper trace). The bottom traces show the integrated electrical activity simultaneously recorded from the opposite preBötC. C, Quantification of the frequency of rhythmic bursts in $\mathrm{Phox}_{2} \mathrm{~b}^{+/+}$and Phox $2 \mathrm{~b}^{27 \mathrm{Ala} /+}$ embryos at pH7.4 and pH 7.2. ns, $p>0.1 . n$, number of slice preparations analyzed. Significant differences in burst frequencies were observed neither between wild-type and mutant embryos nor between the two $\mathrm{pH}$ conditions.

presence of a spontaneous rhythmic activity in both wild-type and mutant slices, whose bursting frequencies did not differ significantly from each other $(6.6 \pm 0.5$ bursts/min for wild types and $6.7 \pm 0.5$ bursts $/ \mathrm{min}$ for Phox $2 b^{27 \mathrm{Ala} /+}$ embryos) (Fig. $6 \mathrm{C}$ ). In contrast to the results obtained for the e-pF, the preBötC network did not respond to low $\mathrm{pH}$ challenge (Fig. $6 \mathrm{C}$ ).

These data extend to a second genetic condition our previous observation in $\mathrm{Egr}^{-1-}$ embryos (Thoby-Brisson et al., 2009) 
showing that the preBötC becomes active independently of a functional e-pF. In intact brainstem preparations, however, the e-pF normally couples to and entrains the preBötC (ThobyBrisson et al., 2009), and this coupling should go awry in the Phox $2 b$ mutants. To investigate this point, we examined phrenic nerve root activity as a measure of the respiratory-like motor outflow that is driven by the preBötC oscillator beyond E15.5 (Thoby-Brisson et al., 2005).

Slowed-down respiratory rhythm and loss of $\mathrm{pH}$ sensitivity in Phox $2 b$ mutant embryos

We thus recorded phrenic nerve root (C4 root) activity in E16.5 brainstem-spinal cord preparations of control and Phox $2 b \mathrm{mu}-$ tant littermates. In control embryos, population recordings from $\mathrm{C} 4$ roots revealed stable rhythmic bursting with occasional skipped cycles and a mean frequency of $6.6 \pm 0.6$ bursts $/ \mathrm{min}$ (Fig. $7 A, E)$. In Phox $2 b^{27 A l a /+}$, Phox $2 b^{\text {lox/lox }} ; L b x 1^{\text {cre/+ }}$, and Phox $2 b^{\text {lox/lox }}$; $E g r 2^{\text {crel+ }}$ embryos, rhythmic phrenic discharges were also detected, but their frequency was markedly reduced, to $2.7 \pm 0.2$ bursts/min in Phox $2 b^{27 \text { Ala/ }+}$ embryos, $2.4 \pm 0.3$ bursts $/ \mathrm{min}$ in Phox $2 b^{\text {lox/lox }} ;$ Egr $^{\text {cre/+ }}$ embryos, and $2.1 \pm 0.4$ bursts/min in Phox $2 b^{\text {lox/lox }} ; L b \times 1^{\text {cre/+ }}$ mutants (Fig. $7 B-D$ ). At birth, a sloweddown respiratory rhythm is seen in Phox $2 b^{27 A l a /+}$ mice as well (Dubreuil et al., 2008). This will likely be the case also for the conditional Phox $2 b$ mutants and may explain their neonatal lethality. There are now four genetic conditions (the Egr2-null and the three Phox $2 b$ mutants), which display absence of a functional e-pF, a slowed-down respiratory rhythm in the embryo and neonatal lethality.

As shown above, the respiratory-like activity that can be recorded in E14.5 hindbrain preparations is markedly accelerated by low $\mathrm{pH}$ challenge in control, but not in any of the mutant embryos. Is this true also at a later stage, once the preBötC has become active and might integrate the inputs from other chemosensitive groups of neurons (Nattie and Li, 2009)? Lowering the $\mathrm{pH}$ from 7.4 to 7.2 resulted in a marked acceleration of the frequency of the $\mathrm{C} 4$ bursts in control preparations (Fig. $7 A, E$ ). In contrast, lowering the $\mathrm{pH}$ was without effect in the mutants (Fig. $7 B-E)$. These results show that in brainstem preparations, Phox2b-expressing cells derived from $\mathrm{Lbxl}^{+}$interneuron precursors in $\mathrm{r} 3$ or $\mathrm{r} 5$ are essential for $\mathrm{CO}_{2} / \mathrm{pH}$ sensitivity. They further demonstrate that at least in in vitro preparations, neurons proposed as $\mathrm{CO}_{2}$ sensors that are not affected by the Phox $2 b$ mutations are unable to restore $\mathrm{CO}_{2} / \mathrm{pH}$ responsiveness.

\section{Discussion}

RTN neurons have been implicated in the generation of respiratory rhythm and $\mathrm{CO}_{2} / \mathrm{pH}$ responsiveness [for review, see Feldman and Del Negro (2006), Onimaru and Homma (2006), Guyenet (2008), and Amiel et al. (2009)]. Here, we have characterized further the development and transcription factor dependence of these neurons. This enabled us to develop new genetic tools to investigate their importance for respiratory-like activity before and survival after birth.

\section{Development of the RTN}

Knowledge of the developmental history of specific groups of respiratory neurons is essential if one wants to generate genetic tools to eliminate them. An important entry point was the discovery of Atoh1 as a selective marker of RTN neurons. In the hindbrain, the Atoh1 transcription factor is known to be expressed in the rhombic lip and its early differentiating progeny (Bermingham et al., 2001; Machold and Fishell, 2005; Wang et al.,
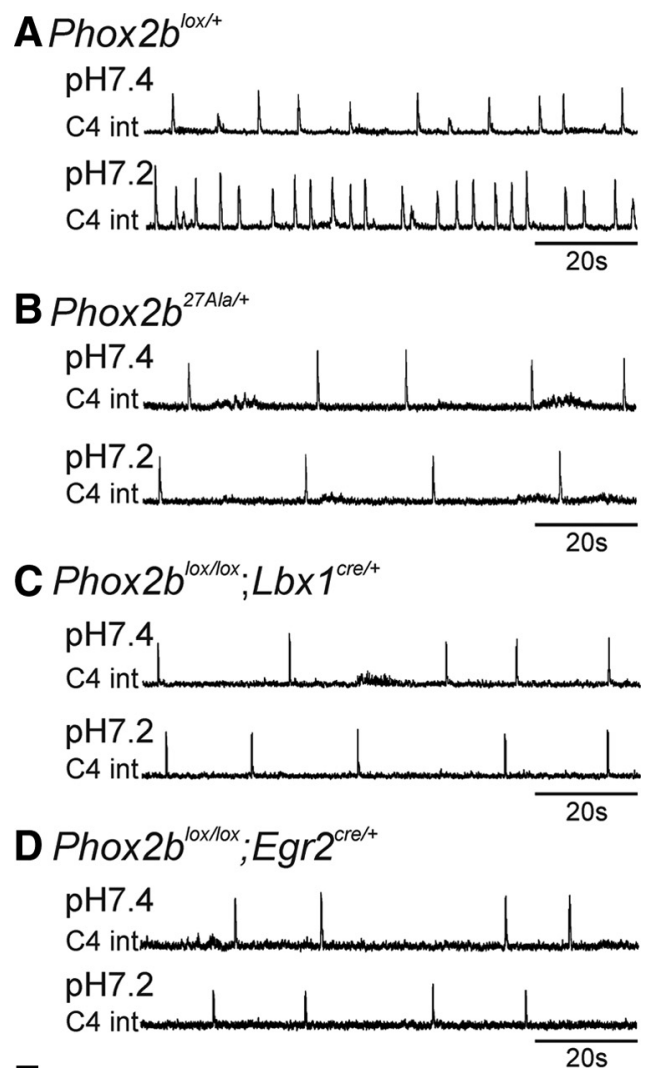

$E$

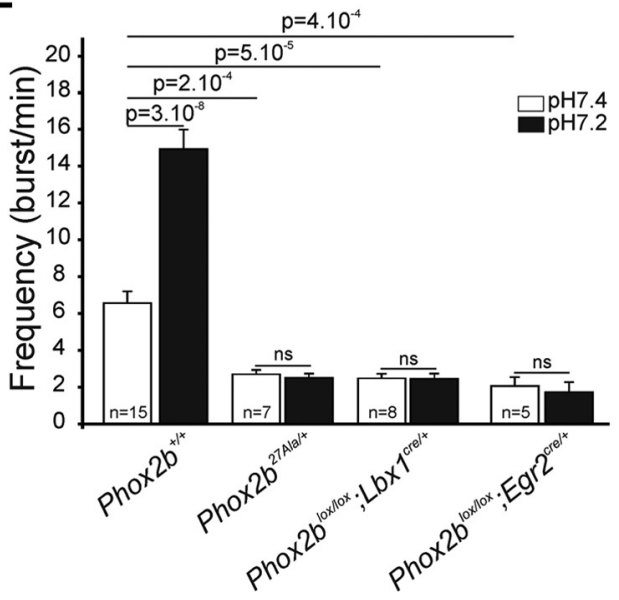

Figure 7. Slowed-down respiratory-like rhythm unresponsive to a low $\mathrm{pH}$ challenge in Phox2b E16.5 mutant embryos. $\boldsymbol{A}$, Integrated phrenic nerve discharges (C4 int) at pH 7.4 (upper trace) and pH 7.2 (lower trace) for a Phox $2 b^{\text {lox/+ }}$ control embryo. $\boldsymbol{B}-\boldsymbol{D}$, Same as $\boldsymbol{A}$

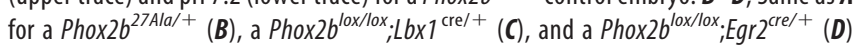
mutant embryo. $\boldsymbol{E}$, Quantification of the burst frequencies for control and mutant embryos as indicated. $p$ values are $3 \times 10^{-8}$ for the effect of lowering the $\mathrm{pH}$ in the controls, and $2 \times 10^{-4}$ to $5 \times 10^{-5}$ for the differences between burst frequencies in control and mutant preparations measured at pH 7.4. ns, $p>0.1 ; n$, number of hindbrain preparations analyzed. The motor output of the respiratory network recorded from $\mathrm{C} 4$ nerve roots was substantially lower in the mutants. In contrast to the marked acceleration seen in control embryos, the frequencies of the rhythmic bursts were not changed by low pH in the mutants.

2005). Our results show that the Atoh1-expressing cells in the ventral medulla represent a separate group of neurons that switch on Atoh1 expression well after they are born and are en route to their final destination. The close topographical relationship between nVII and RTN neurons during their migration and at their final location prompted us to ask whether the latter are attracted 
by the former or otherwise require them for their differentiation. However, in embryos in which the facial precursors do not migrate into r6, neither Atoh1 expression nor the migration of the Phox $2 \mathrm{~b}^{+}$;Atoh $1^{+}$RTN neurons to the pial surface of r6 was perturbed. Conversely, the nVII develops normally when the RTN neurons are missing. Both groups of neurons thus appear to develop independently of each other and may respond to the same guidance cues whose nature remains unknown. Finally, we found that RTN neurons originate from the dorsal interneuron domain in $\mathrm{r} 3$ or $\mathrm{r} 5$, most likely from the $\mathrm{dB} 2$ domain, the only one to express both Phox $2 b$ and $L b x 1$ (Sieber et al., 2007).

\section{Abnormal respiratory rhythmogenesis in Phox $2 b$ mutants}

Phox $2 b^{27 \text { Ala/+ }}$ mutant pups not only lack $\mathrm{CO}_{2}$ responsiveness but also have irregular or slowed-down breathing patterns in normal air (Dubreuil et al., 2008). This could reflect either the dependence of breathing at birth on the tonic drive afforded by the central chemoreflex (Guyenet, 2008) or a more direct role in rhythm generation of the Phox $2 \mathrm{~b}^{+}$neurons compromised in the mutants. Here we show that the spontaneous rhythmic activity detected in the e-pF as early as E14.5 is abrogated in Phox $2 b^{27 A l a /+}$ as well as in the two conditional Phox $2 b$ mutant embryos. This activity is known to be essential for the normal pace of respiratory-like rhythm in late gestation (Thoby-Brisson et al., 2009) and probably also at birth (Jacquin et al., 1996). It is thus likely that the absence of rhythm generation by the e-pF contributes to the abnormal breathing pattern of Phox $2 b^{27 A l a /+}$ and conditional Phox $2 b$ mutants. In all three mutants, a rhythmic activity was present in the facial nucleus and nerve, but it was substantially slower than in wild-type littermates. The origin of this motor rhythm has not been established. It is most likely driven by the still immature preBötC, whose lack of entrainment by the e-pF may cause its slower pace (Thoby-Brisson et al., 2009).

The preBötC, in contrast, became active at E15.5 and appeared to function normally in Phox $2 b^{27 A l a /+}$ slice preparations, demonstrating that its emergence does not require a functional e-pF. However, C4 root recordings in E16.5 brainstem-spinal cord preparations showed that the respiratory-like activity of the phrenic nerve was markedly slower in the three Phox $2 b$ mutants than in control littermates. These results suggest that a functional preBötC is not sufficient by itself to sustain respiratory activity at a normal pace, most probably because it lacks entrainment by a functional e-pF (Thoby-Brisson et al., 2009). In vivo, such a slowed-down breathing pattern may not be compatible with life and may cause the perinatal death of the mutants.

RTN neurons have recently been found to be depleted in Lbx $1^{-/-}$(Pagliardini et al., 2008) and Egr2 ${ }^{-/-}$(Thoby-Brisson et al., 2009) mutant mice. Both mutants also present with complete $\left(\operatorname{Lbx} 1^{-/-}\right)$or partial $\left(E g r 2^{-/-}\right)$neonatal lethality and a slowed-down respiratory rhythm at birth (Jacquin et al., 1996; Pagliardini et al., 2008), again consistent with a role of the RTN in setting the appropriate respiratory pace. However, the massive reorganization of the hindbrain in these mutants may also contribute to the phenotype. For instance, in $L b \times 1^{-1-}$ mutants but not in the Phox $2 b$ mutants, a nonrespiratory slow rhythm normally found throughout the embryonic neural tube at earlier stages persists into late gestation, but was never observed in the Phox $2 b$ mutants.

Although none of the Phox $2 b$ mutations we studied is entirely specific for the RTN, the loss of the Phox $2 \mathrm{~b}^{+}$;Atoh $1^{+}$RTN neurons is the likely cause of the respiratory defect for two reasons. First, while the $E g r 2^{-/-}$and $L b \times 1^{-/-}$mutants may potentially affect any r3/5-derived neuron (Voiculescu et al., 2000) or any neuron derived from the $\mathrm{dB}$ dorsal interneuron domain (Sieber et al., 2007), respectively, the conditional Phox $2 b^{\text {lox/lox }} ; L b \times 1^{\text {cre/+ }}$ and Phox $2 b^{\text {lox/lox }} ; E g r 2^{\text {crel+ }}$ mutations target substantially reduced and only partially overlapping subsets of Phox $2 b$-expressing neurons, but still have the same phenotype. This limits the spectrum of potentially causative neurons to those derived from Phox $2 b$ expressing precursors in the $\mathrm{dB}$ domain of $\mathrm{r} 3 / 5$, including the RTN. Second, although the Phox $2 b^{27 A l a}$ mutation may affect any Phox $2 \mathrm{~b}^{+}$neuron, the only anatomical defect reported was the loss of the RTN (Dubreuil et al., 2008) attesting to the selectivity of its toxic effect. Our data thus strongly suggest that RTN neurons are required for generating a proper respiratory rhythm at late gestation.

\section{Lack of $\mathrm{CO}_{2} / \mathrm{pH}$ responsiveness in Phox $2 \mathrm{~b}$ mutants}

There is still controversy as to the nature and location of the principal neuronal group(s) responsible for central chemosensitivity (Guyenet, 2008; Nattie and Li, 2009). The two prime contenders are medullary raphe 5-HT neurons (Richerson, 2004; Richerson et al., 2005; Hodges et al., 2008) and the RTN (Mulkey et al., 2004; Guyenet et al., 2005). In fact, the RTN and the area where it is located were among the first sites postulated to be important for central chemosensitivity (Schläfke et al., 1975; Smith et al., 1989). The complete unresponsiveness to hypercapnia of Phox $2 b^{27 A l a /+}$ pups, in which the RTN is lost, is consistent with its essential role for $\mathrm{CO}_{2} / \mathrm{pH}$ responsiveness in the perinatal period (Dubreuil et al., 2008), but does not exclude the potential involvement of other sites in which the mutant allele is expressed. Here, we strengthen the case for the RTN as principal chemosensor by showing that central chemosensitivity is lost in two additional mutations that affect the RTN but leave intact other candidate chemosensors. The $\mathrm{CO}_{2} / \mathrm{pH}$ responsiveness of other mutants affecting the RTN was either not explored in $L b \times 1$ mutants (Pagliardini et al., 2008) or found to be preserved in vivo in Egr2 mutants (Jacquin et al., 1996). The latter result contrasts with the complete lack of chemosensitivity observed here in brainstem preparations of $E g r 2^{\text {cre }}$;Phox $2 b^{\text {lox/lox }} \mathrm{em}$ bryos. One possibility is that a subpopulation of RTN neurons generated from Egr2-expressing precursors and thus affected in $E g r 2^{\text {cre/t+}}$;Phox $2 b^{\text {lox/lox }}$ mice does not depend on Egr2 for proper differentiation. Indeed, some of the Egr2 ${ }^{-/-}$(Jacquin et al., 1996), but none of the Egr $2^{\text {cre }}$;Phox $2 b^{\text {lox/lox }}$ mutants survive the neonatal period.

We show that the e-pF oscillator responds to a low $\mathrm{pH}$ challenge with a marked acceleration of its rhythmic activity as soon as it becomes active at $\sim$ E14.5. In contrast, in Phox $2 b^{27 A l a /+}$ and in the two conditional Phox $2 b$ mutants, lowering the $\mathrm{pH}$ neither restores rhythmic activity nor increases the frequency of the motor outflow. The robust response of the phrenic nerve discharge to low $\mathrm{pH}$ at E16.5 is completely lost in the Phox $2 b^{27 \mathrm{Ala} /+}$ and the conditional Phox $2 b$ mutants. Hence, at least in vitro, a number of other candidate chemosensitive sites (Nattie and Li, 2009) are unable to compensate for the loss of RTN neurons. The candidate chemosensors de facto ruled out by our study include those that do not depend on Phox 2b-5-HT neurons (Pattyn et al., 2003) and the preBötC (this paper) - and those that do not derive from $L b \times 1^{+}$precursors (and thus do not lose Phox $2 b$ expression in Phox $2 b^{\text {lox/lox }}$; Lbx $1^{\text {cre/+ }}$ mutants) - noradrenergic cell groups, including the locus ceruleus, and the nucleus of the solitary tract (Sieber et al., 2007; Pagliardini et al., 2008). Our results thus argue in favor of an eminent role for the small number of Phox $2 b^{+}$ sites, including the RTN, which are affected by both conditional mutations. However, the possibility remains that the missing 
Phox2b-expressing neurons are not essential as chemosensors but as obligatory intermediates funneling information from other chemoreceptors to the respiratory rhythm generator. Still, the simplest scenario is that the RTN is the principal $\mathrm{CO}_{2}$ sensor in the perinatal period.

In conclusion, our findings provide genetic evidence for the essential role of Phox $2 b$-expressing neurons in setting the pace of fetal breathing and establishing central chemoreception in a way compatible with survival at birth. The Phox $2 b$ dependence of the RTN together with the discovery of Atoh1 as a new selective marker may provide an entry point for the generation of more refined genetic tools to interfere with the activity of this group of neurons.

\section{References}

Abbott SB, Stornetta RL, Fortuna MG, Depuy SD, West GH, Harris TE, Guyenet PG (2009) Photostimulation of retrotrapezoid nucleus Phox2bexpressing neurons in vivo produces long-lasting activation of breathing in rats. J Neurosci 29:5806-5819.

Akazawa C, Ishibashi M, Shimizu C, Nakanishi S, Kageyama R (1995) A mammalian helix-loop-helix factor structurally related to the product of Drosophila proneural gene atonal is a positive transcriptional regulator expressed in the developing nervous system. J Biol Chem 270:8730-8738.

Amiel J, Laudier B, Attié-Bitach T, Trang H, de Pontual L, Gener B, Trochet D, Etchevers H, Ray P, Simonneau M, Vekemans M, Munnich A, Gaultier C, Lyonnet S (2003) Polyalanine expansion and frame shift mutations of the paired-like homeobox gene $P H O X 2 B$ in congenital central hypoventilation syndrome. Nat Genet 33:459-461.

Amiel J, Dubreuil V, Ramanantsoa N, Fortin G, Gallego J, Brunet J-F, Goridis C (2009) PHOX2B in respiratory control: lessons from congenital central hypoventilation syndrome and its mouse models. Respir Physiol Neurobiol 168:125-132.

Bermingham NA, Hassan BA, Wang VY, Fernandez M, Banfi S, Bellen HJ, Fritzsch B, Zoghbi HY (2001) Proprioceptor pathway development is dependent on Math1. Neuron 30:411-422.

Connelly CA, Ellenberger HH, Feldman JL (1989) Are there serotonergic projections from raphe and retrotrapezoid nuclei to the ventral respiratory group in the rat? Neurosci Lett 105:34-40.

Cream C, Li A, Nattie E (2002) The retrotrapezoid nucleus (RTN): local cytoarchitecture and afferent connections. Respir Physiol Neurobiol 130:121-137.

Dubreuil V, Ramanantsoa N, Trochet D, Vaubourg V, Amiel J, Gallego J, Brunet JF, Goridis C (2008) A human mutation in Phox2b causes lack of CO2 chemosensitivity, fatal central apnea, and specific loss of parafacial neurons. Proc Natl Acad Sci U S A 105:1067-1072.

Feldman JL, Del Negro CA (2006) Looking for inspiration: new perspectives on respiratory rhythm. Nat Rev Neurosci 7:232-242.

Fujiyama T, Yamada M, Terao M, Terashima T, Hioki H, Inoue YU, Inoue T, Masuyama N, Obata K, Yanagawa Y, Kawaguchi Y, Nabeshima Y, Hoshino M (2009) Inhibitory and excitatory subtypes of cochlear nucleus neurons are defined by distinct bHLH transcription factors, Ptfla and Atoh1. Development 136:2049-2058.

Guyenet PG (2008) The 2008 Carl Ludwig Lecture: retrotrapezoid nucleus, CO2 homeostasis, and breathing automaticity. J Appl Physiol 105:404-416.

Guyenet PG, Stornetta RL, Bayliss DA, Mulkey DK (2005) Retrotrapezoid nucleus: a litmus test for the identification of central chemoreceptors. Exp Physiol 90:247-253; discussion 253-257.

Hippenmeyer S, Vrieseling E, Sigrist M, Portmann T, Laengle C, Ladle DR, Arber S (2005) A developmental switch in the response of DRG neurons to ETS transcription factor signaling. PLoS Biol 3:e159.

Hirsch MR, Tiveron M-C, Guillemot F, Brunet J-F, Goridis C (1998) Control of noradrenergic differentiation and Phox2a expression by MASH1 in the central and peripheral nervous system. Development 125:599-608.

Hodges MR, Tattersall GJ, Harris MB, McEvoy SD, Richerson DN, Deneris ES, Johnson RL, Chen ZF, Richerson GB (2008) Defects in breathing and thermoregulation in mice with near-complete absence of central serotonin neurons. J Neurosci 28:2495-2505.

Jacquin TD, Borday V, Schneider-Maunoury S, Topilko P, Ghilini G, Kato F, Charnay P, Champagnat J (1996) Reorganization of pontine rhythmogenic neuronal networks in Krox-20 knockout mice. Neuron 17:747-758.
Lallemand Y, Luria V, Haffner-Krausz R, Lonai P (1998) Maternally expressed PGK-Cre transgene as a tool for early and uniform activation of the Cre site-specific recombinase. Transgenic Res 7:105-112.

Machold R, Fishell G (2005) Math1 is expressed in temporally discrete pools of cerebellar rhombic-lip neural progenitors. Neuron 48:17-24.

Mulkey DK, Stornetta RL, Weston MC, Simmons JR, Parker A, Bayliss DA, Guyenet PG (2004) Respiratory control by ventral surface chemoreceptor neurons in rats. Nat Neurosci 7:1360-1369.

Naiche LA, Papaioannou VE (2007) Cre activity causes widespread apoptosis and lethal anemia during embryonic development. Genesis 45: $768-775$.

Nattie EE, Li A (2002) Substance P-saporin lesion of neurons with NK1 receptors in one chemoreceptor site in rats decreases ventilation and chemosensitivity. J Physiol 544:603-616.

Nattie E, Li A (2009) Central chemoreception is a complex system function that involves multiple brain stem sites. J Appl Physiol 106:1464-1466.

Onimaru H, Homma I (2003) A novel functional neuron group for respiratory rhythm generation in the ventral medulla. J Neurosci 23:1478-1486.

Onimaru H, Homma I (2006) Point:Counterpoint: The parafacial respiratory group (pFRG)/pre-Bötzinger complex (preBötC) is the primary site of respiratory rhythm generation in the mammal. J Appl Physiol 100: 2094-2095

Onimaru H, Ikeda K, Kawakami K (2008) $\mathrm{CO}_{2}$-sensitive preinspiratory neurons of the parafacial respiratory group express Phox $2 \mathrm{~b}$ in the neonatal rat. J Neurosci 28:12845-12850.

Pagliardini S, Ren J, Gray PA, Vandunk C, Gross M, Goulding M, Greer JJ (2008) Central respiratory rhythmogenesis is abnormal in Lbx1deficient mice. J Neurosci 28:11030-11041.

Pattyn A, Morin X, Cremer H, Goridis C, Brunet J-F (1997) Expression and interactions of the two closely related homeobox genes Phox $2 \mathrm{a}$ and Phox2b during neurogenesis. Development 124:4065-4075.

Pattyn A, Morin X, Cremer H, Goridis C, Brunet J-F (1999) The homeobox gene Phox $2 \mathrm{~b}$ is essential for the development of autonomic neural crest derivatives. Nature 399:366-370.

Pattyn A, Hirsch M-R, Goridis C, Brunet J-F (2000) Control of hindbrain motor neuron differentiation by the homeobox gene Phox $2 b$. Development 127:1349-1358.

Pattyn A, Vallstedt A, Dias JM, Samad OA, Krumlauf R, Rijli FM, Brunet J-F, Ericson J (2003) Coordinated temporal and spatial control of motor neuron and serotonergic neuron generation from a common pool of CNS progenitors. Genes Dev 17:729-737.

Richerson GB (2004) Serotonergic neurons as carbon dioxide sensors that maintain pH homeostasis. Nat Rev Neurosci 5:449-461.

Richerson GB, Wang W, Hodges MR, Dohle CI, Diez-Sampedro A (2005) Homing in on the specific phenotype(s) of central respiratory chemoreceptors. Exp Physiol 90:259-266; discussion 266-269.

Rodríguez CI, Buchholz F, Galloway J, Sequerra R, Kasper J, Ayala R, Stewart AF, Dymecki SM (2000) High-efficiency deleter mice show that FLPe is an alternative to Cre-loxP. Nat Genet 25:139-140.

Schläfke ME, Pokorski M, See WR, Prill RK, Loeschcke HH (1975) Chemosensitive neurons on the ventral medullary surface. Bull Physiopathol Respir (Nancy) 11:277-284.

Sieber MA, Storm R, Martinez-de-la-Torre M, Müller T, Wende H, Reuter K, Vasyutina E, Birchmeier C (2007) Lbxl acts as a selector gene in the fate determination of somatosensory and viscerosensory relay neurons in the hindbrain. J Neurosci 27:4902-4909.

Smith JC, Morrison DE, Ellenberger HH, Otto MR, Feldman JL (1989) Brainstem projections to the major respiratory neuron populations in the medulla of the cat. J Comp Neurol 281:69-96.

Spengler CM, Gozal D, Shea SA (2001) Chemoreceptive mechanisms elucidated by studies of congenital central hypoventilation syndrome. Respir Physiol 129:247-255.

Srinivas S, Watanabe T, Lin CS, William CM, Tanabe Y, Jessell TM, Costantini F (2001) Cre reporter strains produced by targeted insertion of EYFP and ECFP into the ROSA26 locus. BMC Dev Biol 1:4.

Stornetta RL, Moreira TS, Takakura AC, Kang BJ, Chang DA, West GH, Brunet J-F, Mulkey DK, Bayliss DA, Guyenet PG (2006) Expression of Phox $2 \mathrm{~b}$ by brainstem neurons involved in chemosensory integration in the adult rat. J Neurosci 26:10305-10314. 
Takakura AC, Moreira TS, Stornetta RL, West GH, Gwilt JM, Guyenet PG (2008) Selective lesion of retrotrapezoid Phox2b-expressing neurons raises the apnoeic threshold in rats. J Physiol 586:2975-2991.

Thoby-Brisson M, Trinh JB, Champagnat J, Fortin G (2005) Emergence of the pre-Bötzinger respiratory rhythm generator in the mouse embryo. J Neurosci 25:4307-4318.

Thoby-Brisson M, Karlén M, Wu N, Charnay P, Champagnat J, Fortin G (2009) Genetic identification of an embryonic parafacial oscillator coupling to the pre-Bötzinger complex. Nat Neurosci 12:1028-1035.

Tiveron M-C, Hirsch M-R, Brunet J-F (1996) The expression pattern of the transcription factor Phox 2 delineates synaptic pathways of the autonomic nervous system. J Neurosci 16:7649-7660.

Varela-Echavarría A, Pfaff SL, Guthrie S (1996) Differential expression of LIM homeobox genes among motor neuron subpopulations in the developing chick brain stem. Mol Cell Neurosci 8:242-257.

Voiculescu O, Charnay P, Schneider-Maunoury S (2000) Expression pat- tern of a Krox-20/Cre knock-in allele in the developing hindbrain, bones, and peripheral nervous system. Genesis 26:123-126.

Voiculescu O, Taillebourg E, Pujades C, Kress C, Buart S, Charnay P, Schneider-Maunoury S (2001) Hindbrain patterning: Krox20 couples segmentation and specification of regional identity. Development 128:4967-4978.

Wang VY, Rose MF, Zoghbi HY (2005) Math1 expression redefines the rhombic lip derivatives and reveals novel lineages within the brainstem and cerebellum. Neuron 48:31-43.

Weese-Mayer DE, Berry-Kravis EM, Marazita ML (2005) In pursuit (and discovery) of a genetic basis for congenital central hypoventilation syndrome. Respir Physiol Neurobiol 149:73-82.

Zechner D, Fujita Y, Hülsken J, Müller T, Walther I, Taketo MM, Crenshaw EB 3rd, Birchmeier W, Birchmeier C (2003) beta-Catenin signals regulate cell growth and the balance between progenitor cell expansion and differentiation in the nervous system. Dev Biol 258:406-418. 